\title{
Lambert-Eaton myasthenic syndrome in a patient with small-cell lung cancer: A case report
}

\author{
RAN-RAN ZHANG ${ }^{1,2^{*}}$, TAO HAN $^{1 *}$, FANG GUO $^{1 *}$, ZHAO-ZHE LIU $^{1 *}$, YA-LING HAN $^{3}$, \\ WEI-CHI CHEN ${ }^{1}$, YONG-YE LIU ${ }^{1}$ and XIAO-DONG XIE ${ }^{1}$

\begin{abstract}
${ }^{1}$ Department of Oncology, Cancer Center, People's Liberation Army General Hospital of Shenyang Military Region, Shenyang, Liaoning 110016; ${ }^{2}$ Department of Oncology, Liaoning Medical University, Jinzhou, Liaoning 121000;

${ }^{3}$ Department of Cardiology, Institute of Cardiovascular Research, People's Liberation Army

General Hospital of Shenyang Military Region, Shenyang, Liaoning 110016, P.R. China
\end{abstract}

Received August 13, 2014; Accepted June 16, 2015

DOI: $10.3892 / \mathrm{ol} .2015 .3473$

\begin{abstract}
Lambert-Eaton myasthenic syndrome (LEMS) is a neuromuscular junction disorder characterized by fluctuating proximal limb muscle weakness, decreased deep tendon reflexes and various autonomic symptoms. LEMS is reportedly the most common neurological paraneoplastic syndrome. This is the case report of a patient with small-cell lung cancer (SCLC) who developed LEMS. A 68-year-old male patient presented with a 6-month history of progressive weakness of the proximal limbs and a 2-month history of xerostomia. The patient was admitted to the Department of Neurology of the People's Liberation Army General Hospital of Shenyang Military Region (Shenyang, China). The symptoms of the patient were not relieved with supportive therapy. Further laboratory tests, electrodiagnostic studies, chest computed tomography and immunohistochemical staining confirmed the diagnosis of LEMS in the presence of SCLC. Following administration of two cycles of rescue chemotherapy with a combination of etoposide and cisplatin, the symptoms of the patient were gradually relieved and, after six cycles of therapy, the primary malignancy completely regressed. In conclusion, a diagnosis of LEMS may lead to the timely detection of SCLC, significantly improving patient prognosis and survival.
\end{abstract}

Correspondence to: Professor Xiao-Dong Xie or Dr Yong-Ye Liu, Department of Oncology, Cancer Center, People's Liberation Army General Hospital of Shenyang Military Region, 83 Wenhua Road, Shenhe, Shenyang, Liaoning 110016, P.R. China

E-mail: doctor_xxd@163.com

E-mail:crzs281@tom.com

*Contributed equally

Key words: Lambert-Eaton myasthenic syndrome, small-cell lung cancer, myasthenia gravis, voltage-gated calcium channel

\section{Introduction}

Paraneoplastic neurological syndromes are usually attributed to autoimmune processes directed against onconeural antigens (1), which are common to cancer cells and the nervous system. Lambert-Eaton myasthenic syndrome (LEMS) is the most common neurological paraneoplastic syndrome (2). LEMS is a neuromuscular junction disorder characterized by fluctuating proximal limb muscle weakness, decreased deep tendon reflexes and various autonomic symptoms. The etiology of LEMS is reduced exocytosis of acetylcholine from nerve endings caused by antibodies directed against voltage-gated calcium channels (VGCCs), increases in the titers of which are observed in $>90 \%$ of patients with LEMS (3).

LEMS is most frequently associated with small-cell lung cancer (SCLC) (4). The estimated annual incidence of LEMS is 10 times lower compared with that of myasthenia gravis (MG), and its frequency in patients with SCLC is 3\% (2). In cases of patients with SCLC and LEMS, a primary diagnosis of LEMS may lead to the identification of early-stage SCLC and a better prognosis compared with that of patients with SCLC alone. However, there is currently no clinical manifestation specific to LEMS or a conclusive test for its diagnosis. The most commonly reported symptom by patients with LEMS is proximal muscle weakness, which is a non-specific symptom that may associated with a number of other diseases (5).

The present case emphasizes that clinicians must remain alert for recognising LEMS, particularly in patients presenting with muscle weakness. This study was approved by the Ethics Committee of the People's Liberation Army General Hospital of Shenyang Military Region (Shenyang, China). The patient signed a written informed consent regarding publication of his medical information.

\section{Case report}

A 68-year-old male patient was admitted to the Department of Neurology of the People's Liberation Army General Hospital of Shenyang Military Region with xerostomia and progressive limb muscle weakness. The weakness was initially noticed in the proximal lower limb musculature, followed by the upper 


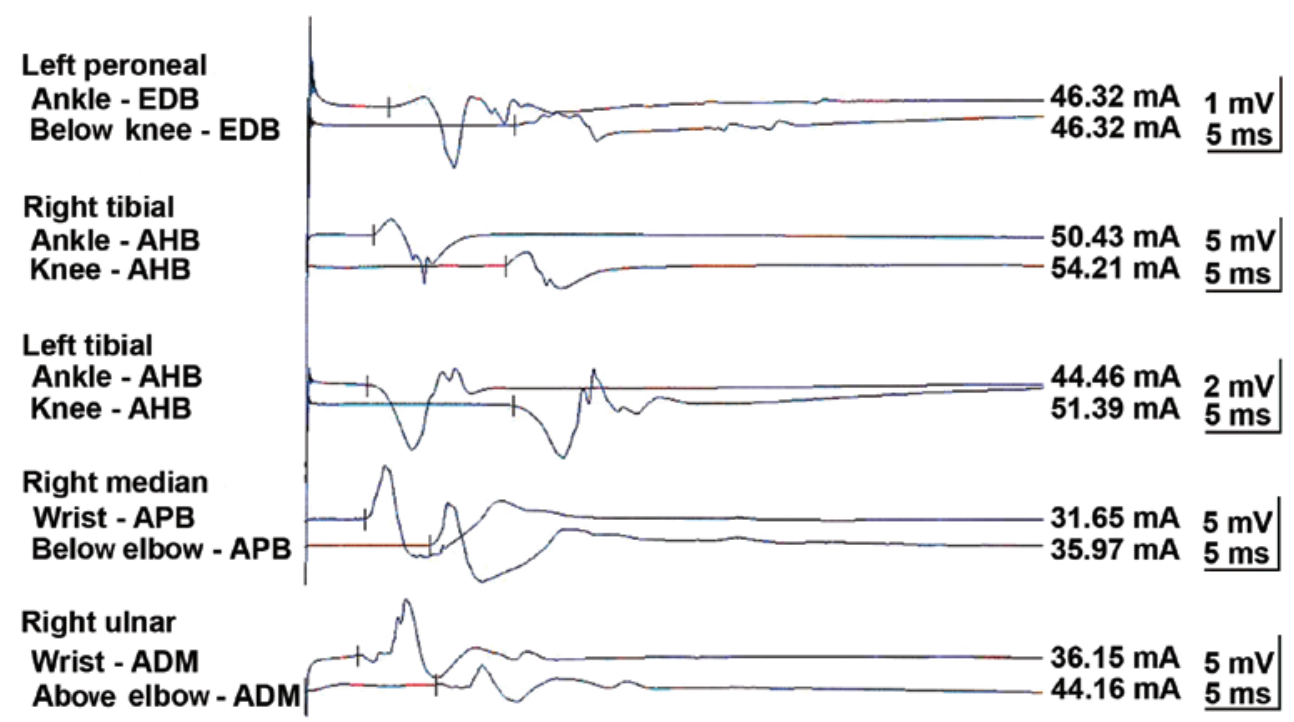

Figure 1. Repetitive nerve stimulation test. EDB, extensor digitorum brevis; AHB, abductor hallucis brevis; APB, abductor pollicis brevis; ADM, abductor digiti minimi.

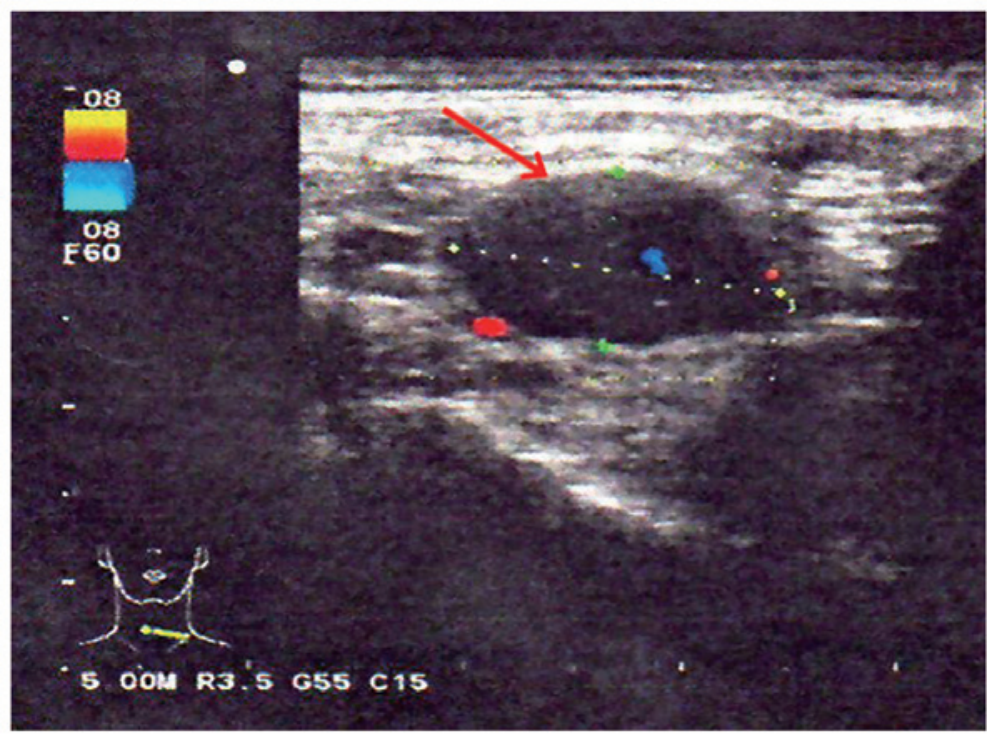

Figure 2. Ultrasound of the right cervical lymph nodes (arrow).

limbs. The patient stated that his proximal limbs were so weak that he could not stand unaided, and that he had lost the ability to live independently. The patient did not report any diurnal variation of weakness and had never experienced persistent cough or hemoptysis. The patient had smoked one pack of cigarettes a day for 50 years. The family history was unremarkable.

A neurological examination revealed proximal muscle weakness and absence of deep tendon reflexes. The cranial nerve, cerebellar and sensory functions were all normal. The laboratory tests revealed hyponatremia and hypochloremia. The electromyogram suggested neurogenic damage (Bilateral abductor muscle and peroneus longus, potential loss of the median nerve, the common peroneal nerve, and mild slowing of the motor conduction velocity of the tibial nerve). A repetitive nerve stimulation test performed on the right median nerve revealed a decremental response to the electrical stimulation.
An experiment with neostigmine supported the presence of postsynaptic membrane lesions. Repeated nerve stimulation of $7 \mathrm{~Hz}$ lead to an increased response, whereas repeated nerve stimulation of $15 \mathrm{~Hz}$ was associated with decreased response (Fig. 1). The findings of magnetic resonance imaging scans of the brain and lumbar vertebra were normal. The preliminary diagnosis of the patient's condition was MG. However, treatment with hypertonic saline and fluid restriction, as well as low-dose corticosteroid therapy for MG, did not improve the symptoms. After referring to several studies in the literature (6-8), LEMS was taken into consideration. The levels of tumor markers, including carcinoembryonic antigen and neuron-specific enolase, were found to be abnormal. An ultrasound of the superficial lymph nodes identified an enlarged lymph node in the right cervical region, sized $1.5 \times 0.9 \mathrm{~cm}$ (Fig. 2). Chest computed tomography (CT) images revealed a $1.6-\mathrm{cm}$ mass in the superior lobe of the right lung, associated 


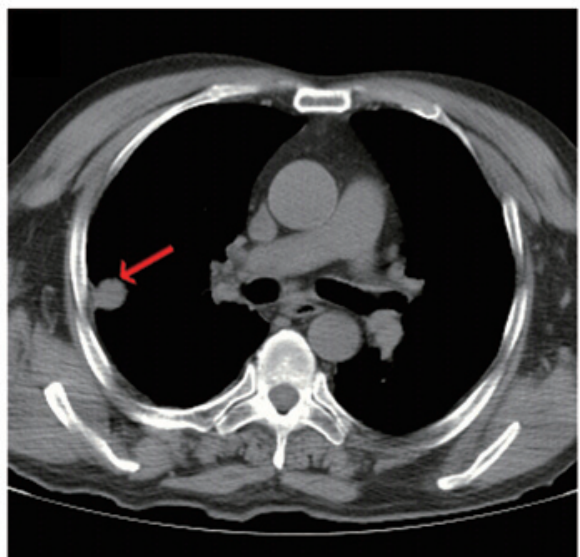

Figure 3. Computed tomography (CT) images. The initial chest CT revealed a mass of $\sim 1.6 \mathrm{~cm}$ located in the right lung (arrow).

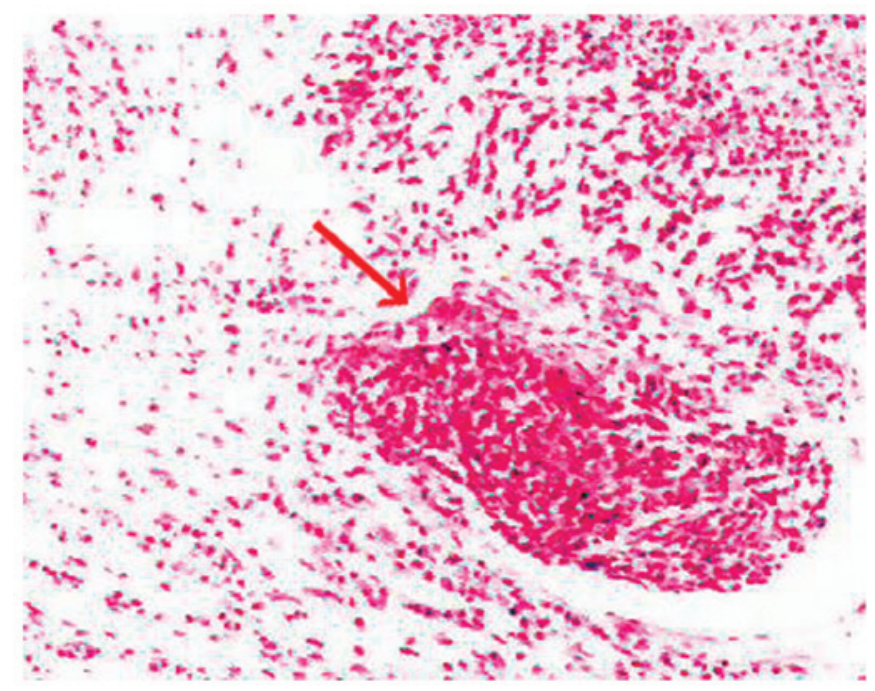

Figure 4. Histopathological examination of the cervical lymph node biopsy specimen, confirming the diagnosis of small-cell lung cancer (arrow) (hematoxylin and eosin staining, magnification x100).

with a small amount of pleural effusion (Fig. 3). A right cervical lymph node biopsy was conducted; on pathological immunohistochemical examination, the lesion was found to be positive for epithelial membrane antigen (+), CD56 (+), thyroid transcription factor-1 $(+)$, creatine phosphokinase $(+)$ and $\mathrm{Ki67}(+++)$ and negative for synaptophysin and leukocyte common antigen. Combined with the findings of the histopathological analysis (hematoxylin and eosin staining; Fig. 4), the diagnosis was eventually confirmed as SCLC combined with LEMS.

In accordance with the National Comprehensive Cancer Network guidelines, the patient was administered etoposide and cisplatin (EP) chemotherapy. After two cycles of EP, the patient exhibited a noticeable improvement in muscular strength (Fig. 5A), was able to walk without assistance, and his ability to live independently was restored. After six cycles of chemotherapy, the primary malignancy disappeared (Fig. 5B) and the sodium and chloride levels returned to normal. The response was evaluated as complete, according to the Response Evaluation Criteria in Solid Tumors 1.0. The
A

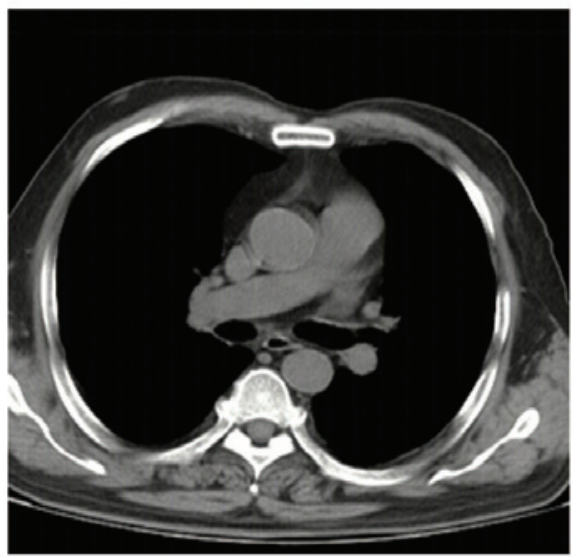

B

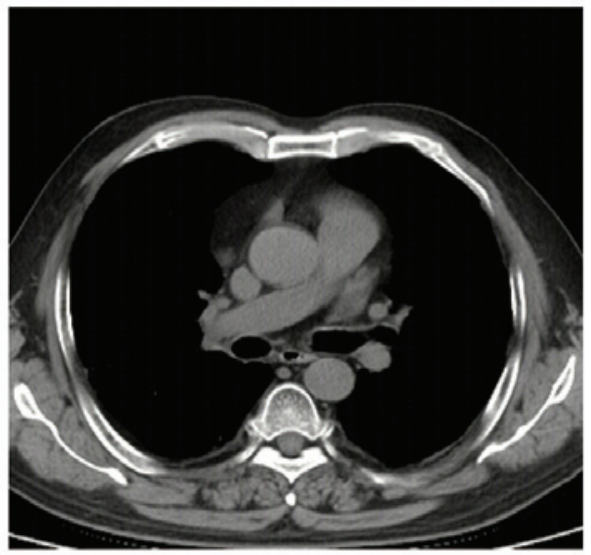

Figure 5. Computed tomography (CT) images. Chest CT images following (A) two and (B) six cycles of rescue chemotherapy.

patient is currently followed up and remains recurrence- and metastasis-free.

\section{Discussion}

In the present case, the main symptom of the patient was the progressive weakness of the limbs, particularly of the proximal muscles. No evident aggravating factors were associated with the onset of the weakness. Thus, when muscle weakness is present, LEMS should be taken into consideration.

Whether the syndrome of inappropriate antidiuretic hormone secretion (SIADH) existed in the present case remains unknown. Since SCLC also causes SIADH (9) and the laboratory tests revealed the presence of hyponatremia and hypochloremia, the muscle weakness may have also been associated with SIADH, as well as LEMS. However, laboratory evidence is required to determine whether the symptoms should be attributed to SIADH rather than LEMS.

The median survival of SCLC patients is 7-8 months. Although the efficiency of first-line chemotherapy is $60 \%$, nearly all the patients recur within 1 year (10). Our patient remains alive for 3 years, without evidence of recurrence or metastasis. Thus, in patients with LEMS, the presence of a paraneoplastic syndrome should be considered. LEMS may be a favorable factor for the prognosis of SCLC $(11,12)$. VGCCs are usually blocked in patients with LEMS. Antibodies against $\mathrm{P} / \mathrm{Q}$ type VGCCs inhibit acetylcholine release from the motor nerve terminals, resulting in muscle weakness (13). 
Anti-VGCC antibodies may play a role in controlling tumor growth, or alternatively, SCLC may cause LEMS to develop slowly (11). However, these possible explanations require further confirmation by a large clinical sample.

In conclusion, a diagnosis of LEMS may lead to the early detection of SCLC, as the clinical symptoms of LEMS usually precede cancer detection (14). Although SCLC is a highly malignant disease with poor prognosis (15), early detection and treatment through diagnosing a paraneoplastic syndrome may significantly improve patient survival.

\section{Acknowledgements}

This study was funded by a grant from the National Research Key Project of the Twelfth Five-Year Plan of P.R. China (no. 2012ZX09303016-002).

\section{References}

1. Kida E, Barcikowska M, Michalska T, et al: Peripheral nervous system alterations in small cell lung cancer. Clinico-pathological study. Neuropatol Pol 30: 43-56, 1992.

2. Sanders DB and Juel VC: The Lambert-Eaton myasthenic syndrome. Handb Clin Neurol 91: 273-283, 2008.

3. Lee JH, Shin HY, Kim SM and Sunwoo IN: A case of Lambert-Eaton myasthenic syndrome with small-cell lung cancer and transient increase in anti-acetylcholine-receptor-binding antibody titer. J Clin Neurol 8: 305-307, 2012.

4. Struthers CS: Lambert-Eaton myasthenic syndrome in small cell lung cancer: nursing implications. Oncol Nurs Forum 21: 677-683; quiz 684-685, 1994.
5. Vincent A, Clover L, Buckley C, et al: Evidence of underdiagnosis of myasthenia gravis in older people. J Neurol Neurosurg Psychiatry 74: 1105-1108, 2003.

6. Sabater L, Höftberger R, Boronat A, Saiz A, Dalmau J and Graus F: Antibody repertoire in paraneoplastic cerebellar degeneration and small cell lung cancer. PLoS One 8: e60438, 2013.

7. Portaro S, Parisi MD, Polizzi A, Ruggieri M, Andreetta F, Bernasconi P, Toscano A and Rodolico C: Long-term follow-up in infantile-onset lambert-eaton myasthenic syndrome. J Child Neurol 29: NP58-61, 2014.

8. Arai $\mathrm{H}$, Inui $\mathrm{K}$, Hashimoto $\mathrm{K}$, et al: Lung adenocarcinoma with Lambert-Eaton myasthenic syndrome indicated by voltage-gated calcium channel: A case report. J Med Case Rep 6: 281, 2012.

9. Fernandez-Torron R, Arcocha J, López-Picazo JM, et al: Isolated dysphagia due to paraneoplastic myasthenic syndrome with anti-P/Q-type voltage-gated calcium-channel and anti-acetylcholine receptor antibodies. Neuromuscul Disord 21: 126-128, 2011.

10. Kuo YH, Lin ZZ, Yang YY, et al: Survival of patients with small cell lung carcinoma in Taiwan. Oncology 82: 19-24, 2012.

11. Ray S, Sonthalia N, Kundu S, et al: Lambert-Eaton myasthenic syndrome and solitary cerebellar metastasis in a patient with occult small-cell lung cancer: a rare experience. BMJ Case Rep 2012, 2012

12. Titulaer MJ, Wirtz PW, Willems LN, et al: Screening for small-cell lung cancer: a follow-up study of patients with Lambert-Eaton myasthenic syndrome. J Clin Oncol 26: 4276-4281, 2008.

13. Kim YI and Neher E: IgG from patients with Lambert-Eaton syndrome blocks voltage-dependent calcium channels. Science 239: 405-408, 1988.

14. Nixdorf DR, Peters E and Lung KE: Clinical presentation and differential diagnosis of nasolabial cyst. J Can Dent Assoc 69: 146-149, 2003.

15. Siegel R, Ward E, Brawley O and Jemal A: Cancer statistics, 2011: the impact of eliminating socioeconomic and racial disparities on premature cancer deaths. CA Cancer J Clin 61: 212-236, 2011. 